\title{
Case Report: Rare Presentation of Invasive Mole Following Term Pregnancy after Assisted Reproduction
}

\author{
S Raguraman ${ }^{1 *}, K_{\text {Muhunthan }}{ }^{1}$ and $P$ Kabileshan ${ }^{2}$ \\ ${ }^{1}$ Senior Lecturer, Department of Obstetrics and Gynaecology, Faculty of Medicine, University of Jaffna, Sri Lanka \\ ${ }^{2}$ Registrar in Obstetrics and Gynaecology, Teaching Hospital Jaffna, Sri Lanka
}

*Corresponding author: S Raguraman, Senior Lecturer, Department of Obstetrics and Gynaecology, Faculty of Medicine, University of Jaffna, Sri Lanka, Tel: 009-477-306-8970

\begin{abstract}
Invasive mole (IM) is one of the Gestational trophoblastic neoplasia lesions, characterized by local invasion of trophoblastic with rare distal spread. It could follow any type of pregnancy and commonly arising from a complete hydatidiform mole (CHM). Pathogenesis of hydatidiform mole due to an underlying oocyte defect. Embryos are examined for triploidy to exclude dispermic fertilization in assisted reproduction technology (ART). Besides, several potential mechanisms and risk factors had been described to explain gestational trophoblastic neoplasia (GTN) following ART. However, ART will not eliminate the risk of development of Gestational trophoblastic disease after several precautions are taken to prevent an embryo with triploidy. This case describes a rare presentation of IM following term delivery after an ART that was successfully managed without distant spread.
\end{abstract}

\section{Keywords}

Invasive mole, Assisted reproductive technology, Intracytoplasmic sperm injection

\section{Introduction}

Gestational trophoblastic disease (GTD) comprises a group of inter related diseases including premalignant and malignant conditions. The malignant diseases of this spectrum are collectively known as gestational trophoblastic neoplasia. GTD can develop after any pregnancy event. Since it can follow any type of pregnancies, the incidence of GTD is difficult to assess [1]. IM is a common manifestation of GTN characterized by the presence of chorionic villi associated with excessive trophoblastic overgrowth and rare distal invasion to the adjacent structures [2]. IM predominantly originates from Complete and Partial hydatidiform and approximately $10-15 \%$ of them may arise from CHM [3].

It is considered that hydatidiform mole develops due to an underlying oocyte defect. The absence of maternal chromosomes in most CHMs and dispermic fertilization in most PHMs and some CHMs supports this [4]. During treatment with ART and especially with intracytoplasmic sperm injection (ICSI), polar bodies and/or blastomeres are examined for triploidy before embryo transfer to exclude dispermic fertilization. Pre-implantation genetic diagnosis (PGD) also play an important role in ART to avoid the transfer and development of an embryo with aneuploidy $[5,6]$.

In this article, we report a case of rare presentation of IM following term delivery after an ART which was successfully managed without distant spread by chemotherapy followed by total hysterectomy and conservation of both ovaries.

\section{Case Report}

A 40-years-old woman with primary subfertility for ten years became pregnant following successful treatment with ART (ICSI). Her pregnancy continued with dichorionic diamniotic twins, complicated with gestational diabetes mellitus and Hypertensive disorder in pregnancy. Due to the above complications, her babies were delivered by an elective caesarean section at 37

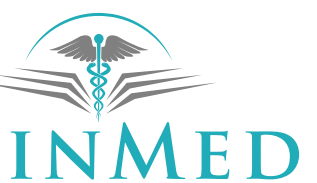

INTERNATIONAL LIBRARY

Citation: Raguraman S, Muhunthan K, Kabileshan P (2021) Case Report: Rare Presentation of Invasive Mole Following Term Pregnancy after Assisted Reproduction. Obstet Gynecol Cases Rev 8:210. doi. org/10.23937/2377-9004/1410210

Accepted: October 04, 2021: Published: October 06, 2021

Copyright: (C) 2021 Raguraman S, et al. This is an open-access article distributed under the terms of the Creative Commons Attribution License, which permits unrestricted use, distribution, and reproduction in any medium, provided the original author and source are credited. 


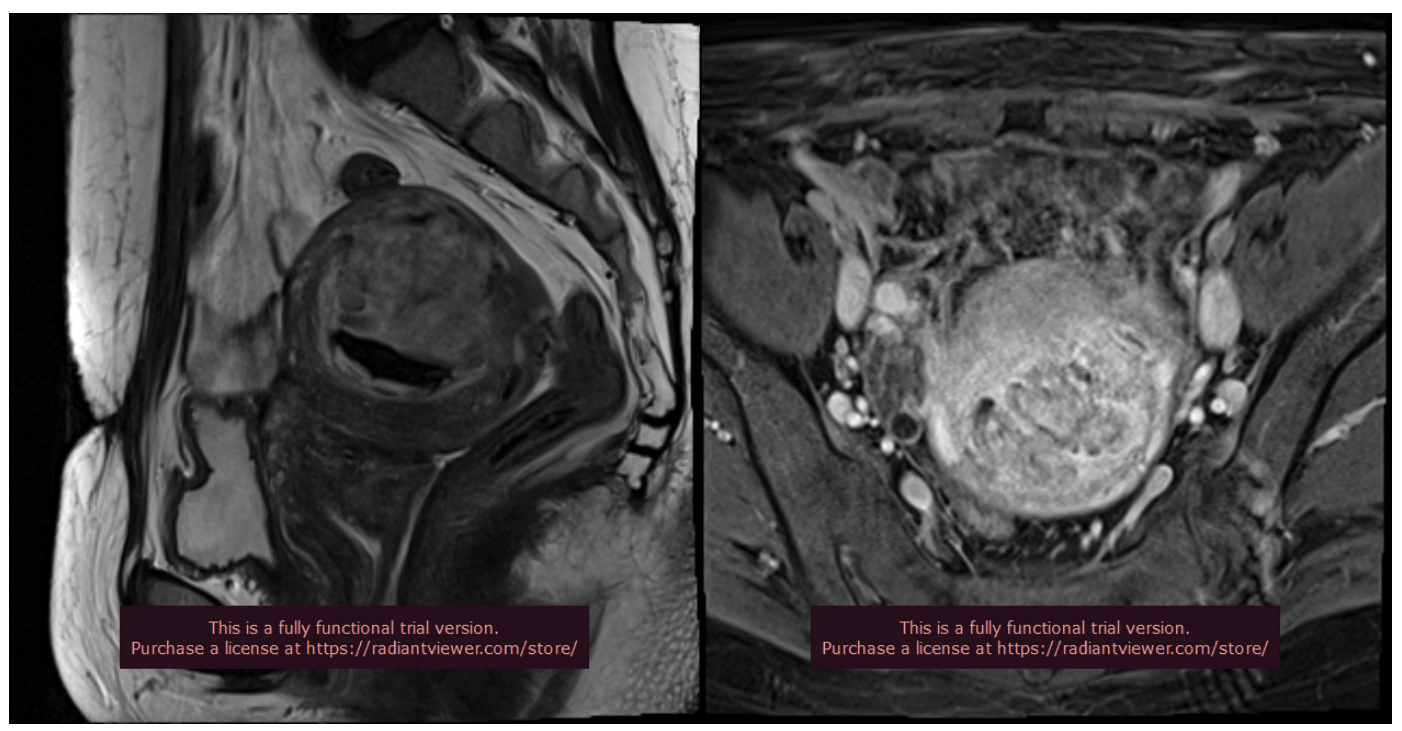

Figure 1 and Figure 2: MRI image of the heterogeneous intense lesion in the uterine cavity with disturbed junctional zone suggestive IM.

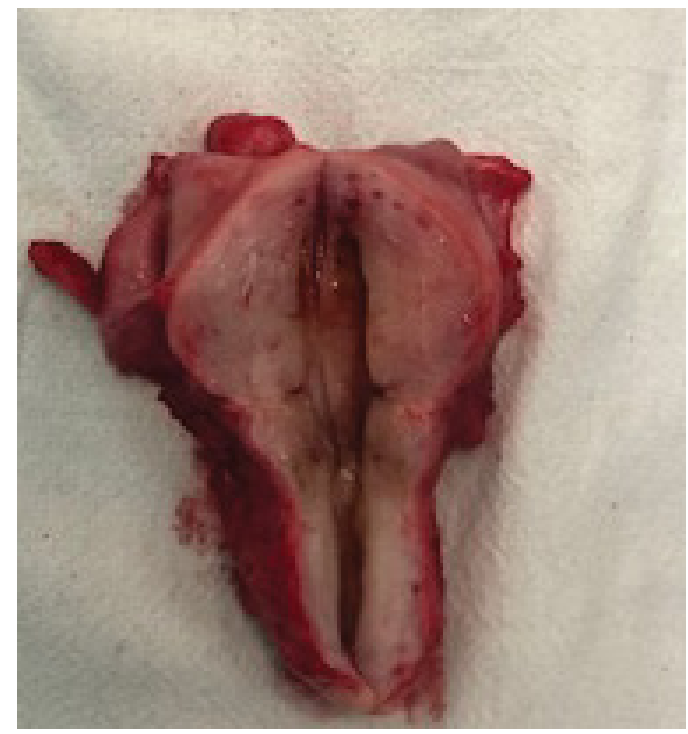

Figure 3: Hysterectomy Specimen without residual molar tissue.

weeks of gestation. Following the caesarian section, she developed a major postpartum haemorrhage treated with uterotonics and blood transfusion and postoperative ICU care. She was discharged on day 10 of postpartum with a good recovery.

She sought medical advice after six months of postpartum with intermittent vaginal bleeding associated with abdominal pain, nausea, vomiting and loss of appetite for two weeks duration. She was sexually inactive for last six months. Her urine hCG was positive during the initial assessment, and serum beta hCG was more than one million units/l. Ultrasound scan was highly suggestive of an invasive mole which was confirmed by MRI chest, abdomen and pelvis without local invasion or distant metastasis (Figure 1 and Figure 2).

She was reviewed by a clinical oncologist and was treated with nine cycles of multi-agent chemotherapy. The serum $\beta$-hCG was $4 \mathrm{IU} / \mathrm{I}$ at the end of the sixth cycle. However, chemotherapy was continued for six weeks after normalization of beta-hCG as initially planned. Based on the patient's age, FIGO score of the disease with no fertility wishes of the patient, the Multidisciplinary team decided to proceed to hysterectomy with conservation of both ovaries to increase the cure rate and prevent the distance spread of this rare and late presentation of IM following ART. Surgery was carried out as planned with uneventful postoperative recovery (Figure 3). Histology suggested a secretory phase of the endometrium with the collection of foamy macrophages with no residual molar tissue. Bilateral tubes and cervix were histologically unremarkable.

\section{Discussion}

GTN is a rare condition and accounts for less than $1 \%$ of all gynaecological malignancies and incidence after a live birth is estimated as 1 in 50,000. IM is characterized by elevated beta hCG with myometrial vascular mass without fetal material, which is commonly confined to the myometrium and rarely spreading to distant tissues $[7,8]$.

Several risk factors have been identified for GTD/ GTN [6]. GTD/GTN following ART is a rare complication despite the widespread practice of advanced fertility treatment. Embryos derived from normally fertilized oocytes are selected for embryo transfer in ART, and divergent pronuclei such as monopronuclei or trimononuclei are strictly avoided to prevent GTN/GTD [9]. Human Fertility and Embryology Authority data also state that the incidences of molar pregnancy following ART pregnancies are much lower than spontaneous conceptions. Further, it shows the overall incidence of molar pregnancy with fresh ICSI was similar to fresh IVF [7]. 
There are several potential mechanisms had been described to explain GTD/GTN following ART. In the ICSI method, triploidy could develop due to digenic (when the extra set of chromosomes is of maternal side) origin or non-extrusion of the second polar body [10]. A few cases reported that triploids were due to diploid sperm and may occur due to retaining the second polar body due to cytoplasmic incompetence [11]. Triploidy has also developed in recure ICSI, where oocytes have been injected following a failed fertilization following conventional IVF. In IVF methods, the occurrence of triploidy is explained by dysfunctional oocyte and polyspermy [12].

IM usually occurs after the evacuation of molar pregnancy and commonly presents as irregular vaginal bleeding with the persistence positive pregnancy test. The usual interval from an antecedent molar pregnancy is less than six months. Serum beta hCG, ultrasound scan and MRI pelvis play a pivotal role in diagnosing IM [13]. IM may be treated with single-agent or multi-agent chemotherapy based on the International Federation of Gynecology and Obstetrics (FIGO) 2000 scoring system for GTN [1].

Our patient presented after six months of term delivery with irregular heavy per vaginal bleeding. She had a history of postpartum haemorrhage, but the review of operative notes did not suggest a possibility of placenta accrete spectrum. Her initial clinical and radiological assessments were highly suggestive of IM. Total abdominal hysterectomy (TAH) with conservation of both ovaries was performed after completion of chemotherapy. Regular follow-up was arranged as she has an increased risk for premature ovarian failure following chemotherapy.

\section{Conclusion}

The woman who develops persistent vaginal bleeding after pregnancy is at risk of GTN irrespective of the birth interval. Besides, ART will not eliminate the risk of the development of GTD/GTN even though several precautions are taken to prevent an embryo with triploidy is transferred. Awareness and index of suspicion are essential to diagnose and facilitate appropriate treatment for rare presentations of GTN following term delivery. Meanwhile, systematic reporting and analysis of GTD/CTN following ART will aid the analysis of the pattern of disease after a different type of ART, prevent further increase in incidence and educate the couple regarding the possibility of GTD before starting ART.

\section{References}

1. Tidy J, Seckl M, Hancock BW (2021) On behalf of the royal college of obstetricians and gynaecologists. Management of Gestational Trophoblastic Disease. BJOG 128: e1-e27.

2. Ilancheran A (1998) Optimal treatment in gestational trophoblastic disease. Ann Acad Med Singap 27: 698-704.

3. Lawrie TA, Alazzam M, Tidy J, Hancock BW, Osborne $R$ (2009) First line chemotherapy in low-risk gestational trophoblastic neoplasia. Cochrane Database Syst Rev 2016: CD007102.

4. Fallahian M (2003) Familial gestational trophoblastic disease. Placenta 24: 797-799.

5. Slim R, Mehio A (2007) The genetics of hydatidiform moles: New lights on an ancient disease. Clin Genet 71: 25-34.

6. Fluker MR, Yuzpe AA (2000) Partial hydatidiform mole following transfer of a cryopreserved-thawed blastocyst. Fertil Steril 74: 828-829.

7. Edwards R, Crow J, Dale S, Macnamee M, Hartshorne $\mathrm{G}$, et al. (1990) Pre-implantation diagnosis and recurrent hydatidiform mole. Lancet 335: 1030-1031.

8. Reubinoff BE, Lewin A, Verner M, Safran A, Schenker JG, et al. (1997) Intra-cytoplasmic sperm injection combined with pre-implantation genetic diagnosis for the prevention of recurrent gestational trophoblastic disease. Hum Reprod 12: 805-808.

9. Nickkho-Amiry M, Horne G, Akhtar M, Mathur, Brison DR (2019) Hydatidiform molar pregnancy following assisted reproduction. J Assist Reprod Genet 36: 667-671.

10. Grossmann M, Calafell JM, Brandy N, Vanrell JA, Rubio C, et al. (1997) Origin of tri-pronucleate zygotes after intracytoplasmic sperm injection. Hum Reprod 12: 27622765.

11. Macas E, Imthurn B, Keller PJ (2001) Increased incidence of numerical chromosome abnormalities in spermatozoa injected into human oocytes by ICSI. Hum Reprod 16: 115120.

12. Rosen M, Shen S, Dobson AT, Fujimoto VY, McCulloch CE, et al. (2006) Triploidy formation after intracytoplasmic sperm injection may be a surrogate marker for implantation. Fertil Steril 85: 384-390.

13. El-agwany AS (2014) Uterine intramural persistent mole: A case report following molar pregnancy evacuation with arteriovenous malformation. Egypt J Radiol Nucl Med 45: 1291-1294. 